\title{
Infliximabe no Tratamento Inicial da Retocolite Ulcerativa Moderada e Grave. Terapia Top Down: Relato Preliminar
}

\author{
Infliximab: First Line Therapy in Severe Ulcerative Colitis. \\ Preliminary Report
}

\author{
FABIO VIEIRATEIXEIRA ${ }^{1}$; ROGÉRIO SAAD-HOSSNE ${ }^{2}$; MAURÍCIO RAMPINELLI CARPI ${ }^{3}$; \\ ANACLAUDIA DE AQUINO TEIXEIRA ${ }^{3}$; PAULO TEIXEIRA JÚNIOR ${ }^{3}$
}

\author{
${ }^{1}$ Especialista em Coloproctologia pela Sociedade Brasileira de Coloproctologia, Professor Voluntário do \\ Departamento de Cirurgia e Ortopedia e do Ambulatório de Doenças Inflamatórias Intestinais da Faculdade de \\ Medicina - UNESP Botucatu e Médico da UNIGASTRO, Marília; ${ }^{2}$ Professor Assistente Doutor do Departamento de \\ Cirurgia e Ortopedia - UNESP Botucatu. ${ }^{3}$ Médico(a) da UNIGASTRO, Marília - São Paulo - Brasil.
}

\begin{abstract}
TEIXEIRA FV; SAAD-HOSSNE R; CAPRI MR; TEIXEIRAACA; TEIXEIRA JÚNIOR P. Infliximabe no Tratamento Inicial da Retocolite Ulcerativa Moderada e Grave. Terapia Top Down: Relato Preliminar de 2 Casos. Rev bras Coloproct, 2008;28(3): 289-293.

RESUMO: Objetivo: A primeira opção para o tratamento da retocolite ulcerativa inespecífica (RCU) se resume em: salicilatos (mesalazina e sulfassalazina) nos casos leves, e corticóides nos casos mais graves. Recentemente, em novembro de 2006 , o Ministério da Saúde aprovou o infliximabe (REMICADE ${ }^{\circledR}$ - Mantecorp - Brasil), anticorpo monoclonal murino contra o fator de necrose tumoral / TNF, para o tratamento da RCU (Escore de Mayo acima de 7). Entretanto, a droga somente tem sido usada como última opção naqueles pacientes refratários ao tratamento convencional ou que sejam corticodependentes. $O$ objetivo desse estudo foi relatar o uso do infliximabe como primeira opção para o tratamento de dois pacientes portadores de RCU tratados no Hospital UNIMAR e no ambulatório de Doenças Inflamatórias Intestinais da FME-UNIMAR. Métodos e resultados: Paciente 1: AZF, 52 anos, sexo feminino, foi diagnosticada primeiramente com o RCU baseado na história e no exame clínico; Colonoscopia compatível com pancolite e biópsia positiva para RCU (microabscesso de cripta). O escore de Mayo era 10 (escala: 0 a 12 / 0 = assintomática 12 colite grave). Recebeu infusão intravenosa de infliximabe (5mg / $\mathrm{Kg}$ de peso) nas semanas $0,2,6$ e 14 (indução). Posteriormente, foi introduzido mesalazina 4,5 g/dia como terapia de manutenção. Observou-se resposta clínica significativa baseada no escore de Mayo analisado após as doses de indução (escore de 10 para 7 - redução de 7 pontos) Atualmente, a paciente está assintomática. Paciente 2: MLA, 45 anos, sexo feminino, com queixa de diarréia muco-sanguinolenta; colonoscopia compatível com colite de cólon esquerdo; biópsia positiva; escore de Mayo de 9 pontos. A paciente recebeu infliximabe EV (5mg/Kg de peso) nas semanas 0, 2, 6 e 14. Após a indução foi introduzido a mesalazina 4.2 g/dia. Atualmente, a paciente está assintomática com escore de Mayo de 2 pontos. Conclusões: Após extensa revisão bibliográfica, acreditamos que esse seja o primeiro relato em língua Portuguesa do uso de um agente biológico como primeira opção no tratamento da RCU. No entanto, mesmo com o sucesso obtido, esse relato inicial deve ser analisado com cautela. A pergunta se o uso do infliximabe seria a melhor opção na terapia inicial das formas graves da $\mathrm{RCU}$, ainda precisa ser respondida por meio de estudos randomizados e controlados.
\end{abstract}

Descritores: Retocolite ulcerativa inespecífica, tratamento, infliximabe, Terapia TOP DOWN.

Trabalho realizado no Serviço de Endoscopia e Cirurgia do Aparelho Digestivo e Coloproctologia - UNIGASTRO / Hospital UNIMAR, Marilia, São Paulo - Brasil. 
Infliximabe no Tratamento Inicial da Retocolite Ulcerativa Moderada e Grave. Terapia Top Down: Relato Preliminar de 2 Casos Fabio Vieira Teixeira e Cols.
O tratamento da retocolite ulcerativa inespecífica (RCU) normalmente está baseado no uso de salicilatos (sulfassalazina, mesalazina), corticóides e imunossupressores. Cerca de $10 \%$ dos pacientes são portadores das formas graves da doença, que requerem hospitalização e tratamento com corticóides e drogas imunossupressoras administradas por via endovenosa. Entretanto, mesmo assim, cerca de 20 a $40 \%$ desses pacientes ainda são refratários aos tratamentos mais agressivos, e são submetidos a colectomia total e confecção de bolsa ileal para controle da doença. ${ }^{1-8}$

O uso dos agentes biológicos com o infliximabe vem sendo empregado no tratamento da RCU desde 2001. Estudos randomizados e controlados mostraram o benefício do uso do infliximabe nos casos de RCU moderada e grave tanto na indução da remissão quanto na diminuição no número de colectomias em pacientes com RCU. ${ }^{1-2}$

Em novembro de 2006, o Ministério da Saúde do Brasil aprovou o uso do infliximabe nos casos graves de RCU que sejam refratários ao uso de medicações convencionais. Baseado no tratamento top down da doença de Crohn (DC), acreditamos que talvez, em casos graves, o infliximabe poderia ser usado como primeira escolha no tratamento da RCU. E, talvez, poderíamos evitar a evolução da doença para formas mais graves, e conseqüentemente a necessidade de colectomia. Portanto, assim como proposto para a DC, acreditamos que a terapia top down poderia modificar a história natural da RCU. Os objetivos deste trabalho são reportar o uso do infliximabe como terapia inicial no tratamento de 2 pacientes portadores de RCU moderada e grave, e avaliar os resultados clínicos obtidos após o tratamento. ${ }^{3,5}$

\section{MATERIAL E METODOS}

A partir de uma análise inicial de dois pacientes submetidos ao tratamento "terapia top down" COM INFLIXIMAB, para pacientes com RCUI moderada e grave

Paciente 1: Paciente, AZF, 52 anos, sexo feminino, deu entrada no Hospital UNIMAR com quadro de diarréia muco-sanguinolenta fazia 3 meses. Foi submetida a colonoscopia com diagnóstico macroscópico compatível com pancolite; a biópsia revelou microabscesso de cripta (Figura 1). O escore de Mayo era 10 (escala: 0 a $12 / 0=$ assintomática 12 colite grave). Após discutir com a paciente as opções terapêuticas disponíveis e os efeitos colaterais dos corticosteróides, a paciente optou em ser submetida à terapia biológica como primeira escolha. Recebeu infusão intravenosa de infliximabe ( $5 \mathrm{mg} / \mathrm{Kg}$ de peso) nas semanas 0, 2, 6 (indução) e uma dose de manutenção na semana 14. Posteriormente, foi introduzido mesalazina 4,5 g/dia como terapia de manutenção. Observou-se resposta clínica significativa baseada no escore de Mayo analisado após as doses de indução (escore de 10 para 7 - redução de 7 pontos) e na colonoscopia (Figura 2). Atualmente, a paciente está assintomática fazendo uso de $3,0 \mathrm{~g} /$ dia de mesalazina.

Paciente 2: MLA, 45 anos, sexo feminino, com queixa de diarréia muco-sanguinolenta; colonoscopia

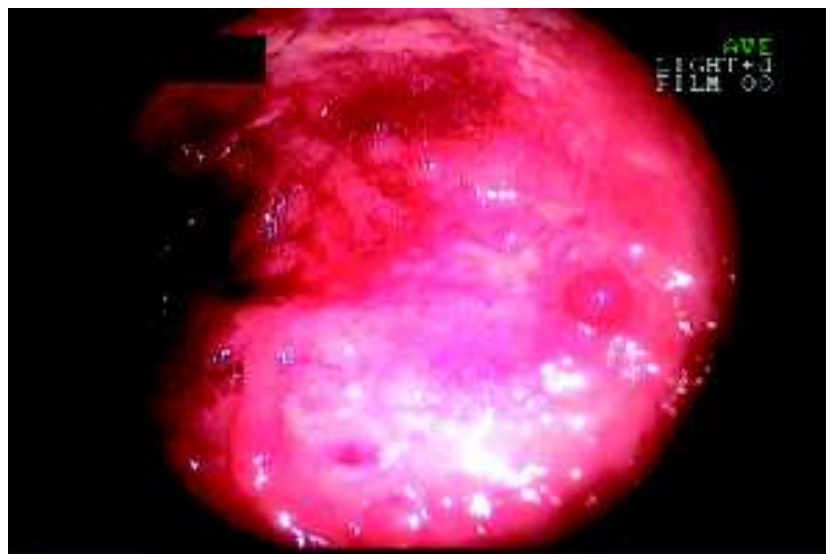

Figura 1 - Colonoscopia em paciente com sintomas de RCU: observa-se presença de processo inflamatório intenso da mucosa cólica, friabilidade da parede ao toque do aparelho e inúmeros pseudopólipos. Sub-escore de Mayo 3.

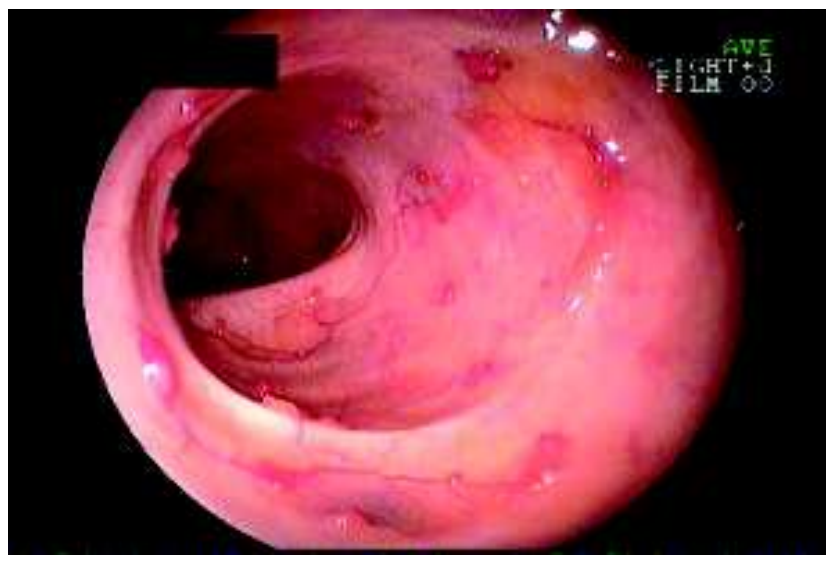

Figura 2 - Colonoscopia em paciente com RCU submetido à terapia top down: observa-se cicatrização da mucosa cólica e a presença de pseudopólipos. Sub-escore de Mayo 0. 
compatível com colite de cólon esquerdo; biópsia positiva; escore de Mayo de 9 pontos. A paciente recebeu infliximabe EV (5mg/Kg de peso) nas semanas 0, 2, 6 e 14. Após a indução foi introduzido a mesalazina 4.2 $\mathrm{g} /$ dia. Atualmente, a paciente está assintomática com escore de Mayo de 2 pontos fazendo uso de 2,4 g de mesalazina por dia.

\section{DISCUSSÃO}

Como se sabe, o tratamento das doenças inflamatórias intestinais (DII) tem por objetivo principal induzir a remissão da doença, e conseqüentemente melhorar a qualidade de vida dos pacientes uma vez que não se pode obter a cura.

Na maioria dos consensos mundiais, a abordagem terapêutica das DII deve ser de forma gradativa, primeiramente iniciando-se com drogas como corticóides e salicilatos, e posteriormente introduzindose os imunomoduladores como a azatioprina e o metrotexato. $\mathrm{O}$ uso dos agentes biológicos como o infliximabe, deve ser indicado como terapia de última escolha nos casos refratários. A esse tipo de estratégia terapêutica denominou-se o nome de step up. ${ }^{6}$

$\mathrm{O}$ termo terapia top down foi primeiramente empregado pelos reumatologistas no tratamento da artrite reumatóide. ${ }^{9} \mathrm{O}$ significado real seria o emprego inicial, como primeira escolha, de drogas até então tidas como de última escolha no tratamento - drogas reservadas para os casos graves da doença, refratários a qualquer outro tipo de medicação. Pacientes portadores de artrite reumatóide que receberam precocemente imunossupressores, e agentes biológicos tiveram mais rápida diminuição na atividade inflamatória da doença e interrupção das lesões articulares. ${ }^{9}$

Baseados em experiência prévia obtida na reumatologia, Hommes e colaboradores, pela primeira vez, delinearam um estudo comparando terapia top down a terapia step up em pacientes portadores de DC moderada e grave. Foram randomizados 133 pacientes com IDAC (índice de atividade da doença de Crohn) $>220$ pontos, com diagnóstico obtido em um intervalo inferior a 4 anos e que nunca tinham recebido tratamento anterior. Foram criados 2 grupos: aqueles submetidos à terapia top down (infliximabe + azatioprina) e aqueles que se submeteram à terapia convencional denominada step up (budesonida ou prednisona). Os autores observaram que $81 \%$ dos pacientes alocados no grupo top down obtiveram re- missão da doença comparados a $73 \%$ dos pacientes que receberam terapia step up $(\mathrm{p}<0,003)$. Concluíram que o uso da terapia top down é superior a terapia step up na indução da remissão em pacientes portadores de DC moderada e grave. ${ }^{3}$ Porém, esse é o único estudo randomizado publicado na literatura mundial que comparou os dois tipos de abordagem terapêutica: top down e step up. ${ }^{3,6}$ Em breve, a resposta se abordagem top down é mais efetiva se comparada a tradicional step up poderá ser respondida por um estudo multicêntrico conhecido por SONIC (study of immunomodulator naive patients in Crohn's disease).

Hoje existe evidência científica de que o uso precoce de infliximabe em portadores de RCU, poderia induzir a cicatrização da mucosa intestinal, e conseqüentemente a diminuição da hospitalização e necessidade de colectomia. ${ }^{1-2}$ No entanto, não está claro que a cicatrização da mucosa intestinal, por si só, esteja relacionada com a mudança da história natural da DC ou da RCU.

Até o presente momento, não existem estudos randomizados e controlados que compararam o uso da terapia step up com a terapia top down no tratamento da RCU. Recentemente, Castro Fernadéz e colaboradores, relataram o sucesso do uso do infliximabe em um caso de megacólon tóxico refratário ao uso de corticóides. ${ }^{5}$ Concluíram que não se deve indicar o uso do infliximabe como tratamento de escolha do megacólon tóxico, porém, o uso de um agente biológico deveria ser considerado na RCU moderada e grave após 3 a 4 dias de uso sem sucesso clínico de corticóides endovenosos. Nos 2 casos aqui descritos, também observamos uma melhora rápida dos pacientes após a infusão inicial de infliximabe $5 \mathrm{~m} / \mathrm{kg}$. Entretanto, nenhum de nossos pacientes recebeu tratamento com corticóides previamente.

No nosso conhecimento, esse é o primeiro relato na literatura do uso de um agente biológico como terapia inicial no tratamento da RCU. Em nossa opinião, o uso da terapia top down nos casos de RCU grave se justifica, uma vez que cerca de $40 \%$ dos pacientes não respondem a terapia inicial com corticóides. ${ }^{1}$ Desses, cerca de $20 \%$ a $60 \%$ necessitarão de algum tipo de operação em um intervalo de 3 meses. ${ }^{1-2} \mathrm{Ba}-$ seados na experiência prévia de pacientes reumatológicos portadores de artrite reumatóide, bem como no trabalho publicado por Hommes e colaboradores, em nossa opinião, não há dúvida que o uso pre- 
Rev bras Coloproct Julho/Setembro, 2008
Infliximabe no Tratamento Inicial da Retocolite Ulcerativa Moderada e Grave. Terapia Top Down: Relato Preliminar de 2 Casos Fabio Vieira Teixeira e Cols.
Vol. 28 coce de imunossupressores e agentes biológicos, poderia ser indicado nos pacientes com RCU moderada e grave. No entanto, por outro lado, infelizmente não existem marcadores clínicos, endoscópicos, radiológicos ou bioquímicos que podem predizer quais pacientes seriam os maiores beneficiados com a terapia top down.

Esse relato inicial é muito pouco para podermos tirar conclusões definitivas se a terapia top down é a melhor abordagem terapêutica da RCU. Apesar do sucesso obtido nesses 2 casos por nós descritos, qualquer conclusão final poderia ser precipitada. Em nossa opinião, estudos clínicos randomizados e controlados poderiam responder se o uso de uma abordagem terapêutica top down é mais efetiva que a convencional no tratamento da RCU moderada e grave, principalmente na indução e manutenção da remissão.

\begin{abstract}
The first option for the treatment of UC is both: salicylates or corticoids. Recently, in late November of 2006, the Brazilian Ministry of Health has approved infliximab (Remicade $\odot$, Mantecorp, Brazil) to treat ulcerative colitis. We report the use of infliximab as a first option for the treatment of two patients with severe ulcerative colitis. Case report: Patient 1: AZF, 52 years-old, female, was first diagnosed with UC after history and clinical examination; colonoscopy showed pancolitis with positive biopsy (crypt microabscess). Her Mayo score was 10 (range: 0 to 12 / asymptomatic to severe colitis). She received intra venous infusion of infliximab at a dose of $5 \mathrm{mg} / \mathrm{Kg}$ of body weigh at week $0,2,6$ and 14 . Then, patient was given mesalazine $4.5 \mathrm{~g}$ / day for maintenance therapy. Clinical response was defined as a decreased from baseline in the total Mayo score of at least 3 points. At present, patient is asymptomatic with Mayo score of 3 one moth after the last dose of infliximab. Patient 2: MLA, 45 years-old, female was first diagnosed with bloody diarrhea; colonoscopy showed left colitis and the biopsy was positive for ulcerative colitis. Her Mayo score was 9 . She was offered and accepted the step down treatment. She was given infliximab $5 \mathrm{mg} /$ $\mathrm{Kg}$ of body weight at week $0,2,6$ and 14. After initial treatment with infliximab, she received mesalazine $4.2 \mathrm{~g} / \mathrm{day}$. At present, she is asymptomatic with Mayo score of 2 eighteen days after the last dose of infliximab. At our knowledge, this is the first Brazilian report of the use of infliximab as fist-line therapy in ulcerative colitis. Few days after the begging of the infusion, an impressive clinical and colonoscopy improvement was seen in these two patients. Recently, it has been reported the use of infliximab as first-line therapy in pediatric Crohn disease. Infliximab could be a good option in cases of moderate and severe UC to avoid the side effects of the use of high doses of corticoids in patients with moderate and severe UC. However, the question if step-down therapy in ulcerative colitis is better then conventional therapy with salicylates and corticoids needs to be answered by randomized trials.
\end{abstract}

Key words: Ulcerative colitis, Treatment, Infliximab, Top Down therapy.

\section{REFERÊNCIAS}

1. Lees CW, Heys D, Ho GT, Noble CL, Shand AG, Mowat C, Boulton-Jones R, Williams A, Church N, Satsangi J, Arnott ID; Scottish Society of Gastroenterology Infliximab Group. retrospective analysis of the efficacy and safety of infliximab as rescue therapy in acute severe ulcerative colitis. Aliment Pharmacol Ther. 2007;26(3):411-9.

2. Järnerot G, Hertervig E, Friis-Liby I, Blomquist L, Karlén P, Grännö C, Vilien M, Ström M, Danielsson A, Verbaan H, Hellström PM, Magnuson A, Curman B.Infliximab as rescue therapy in severe to moderately severe ulcerative colitis: a randomized, placebo-controlled study. Gastroenterology. 2005;128(7):1805-11.

3. Hommes D, Baert F, van Assche G, Caenepeel F, Vergauwe P, H. T, De Vos M, van Deventer S, Stitt L, Rutgeerts P, Feagan B, D'Haens G. The ideal management of Crohns Disease: top down versus step up strategies, a randomized controlled trial. Gastroenterology 2006;130:A-108-109 Abstract 749.

4. Hyams J, Crandall W, Kugathasan S, Griffiths A, Olson A, Johanns J, Liu G, Travers S, Heuschkel R, Markowitz J, Cohen S, Winter H, Veereman-Wauters G, Ferry G, Baldassano R, Group RS. Induction and maintenance infliximab therapy for the treatment of moderate-to-severe Crohn's disease in children.Gastroenterology 2007;132:863-73

5. Castro Fernández M, García Romero D, Sánchez Muñoz D, Grande L, Larraona JL. Severe ulcerative colitis, with toxic megacolon, resolved with infliximab therapy. Rev Esp Enferm Dig. 2007;99(7):426-7

6. Oldenburg B, Hommes D. Biological therapies in inflammatory bowel disease: top-down or bottom-up? Curr Opin Gastroenterol. 2007;23(4):395-9

7. Hanauer SB, Feagan BG, Lichtenstein GR, Mayer LF, Schreiber S, Colombel JF, Rachmilewitz D, Wolf DC, Olson A, Bao W, 
Rutgeerts P; ACCENT I Study Group. Maintenance infliximab for Crohn's disease: the ACCENT I randomised trial. Lancet. 2002;359(9317):1541-9

8. Rutgeerts P, Sandborn WJ, Feagan BG, Reinisch W, Olson A, Johanns J, Travers S, Rachmilewitz D, Hanauer SB, Lichtenstein GR, de Villiers WJ, Present D, Sands BE, Colombel JF.Infliximab for induction and maintenance therapy for ulcerative colitis. N Engl J Med. 2005;353(23):2462-76

9. Breedveld FC, Emery P, Keystone E, Patel K, Furst DE, Kalden JR, St Clair EW, Weisman M, Smolen J, Lipsky PE, Maini RN. Infliximab in active early rheumatoid arthritis. Ann Rheum Dis. 2004;63(2):149-55.

\section{Endereço para correspondência:}

PROF. DR. FABIO V. TEIXEIRA - UNIGASTRO

Rua Dr. Próspero Cecílio Coimbra, 80 - Cidade Universitária

Marilia - São Paulo - Brasil

CEP 17525-160

TEL / FAX +55 14 2105-4562

E-mail fabioteixeira@unimedmarilia.com.br 